\title{
MÉDIAS CLIMÁTICAS PARA OS ÚLTIMOS CICLOS NODAIS LUNARES E PERÍODOS DE MANCHAS SOLARES: AVALIAÇÕES EM CIDADES PERNAMBUCANAS
}

\author{
LOPES, Breno ${ }^{1}$ \\ SOUSA, José Sebastião Costa de ${ }^{2}$ \\ ROCHA, Marlon Gomes da ${ }^{3}$ \\ LOPES, Iug ${ }^{4}$ \\ LEAL, Brauliro Gonçalves ${ }^{5}$
}

\begin{abstract}
RESUMO: As alterações dos fenômenos climáticos no mundo têm sido intensamente discutidas nos últimos anos, gerando inclusive compreensões divergentes do real cenário contemporâneo. Neste sentido objetivou-se com o presente trabalho avaliar a influência dos ciclos nodais lunares e de períodos de atividades solares no comportamento climatológico de cidades pernambucanas. $\mathrm{O}$ trabalho foi realizado com dados de precipitação e temperatura para as estações de Petrolina-PE e Recife-PE, obtidos junto ao Instituto Nacional de Meteorologia (INMET), para o período de 1961 a 2018. Avaliaram-se períodos de 10 anos correspondentes aos ciclos nodais lunares e períodos de 11 anos para os ciclos solares de Schwabe, observando a variação das variáveis descritas. As avaliações foram realizadas através de planilhas eletrônicas e geração de regressões lineares e linhas de tendência. Observou-se que os ciclos não apresentaram influência direta nas variáveis estudadas e que houve acréscimos nas temperaturas e decréscimos de pluviosidade. Sendo que a maioria das tendências, nos ciclos, apresentou variações próximas à tendência da base total de dados.
\end{abstract}

Palavras-chave: Petrolina. Recife. Temperatura. Precipitação. Schwabe.

\section{CLIMATIC AVERAGES FOR THE LAST LUNAR NODAL CYCLES AND SUNSPOT PERIODS: EVALUATIONS IN PERNAMBUCO CITIES}

SUMMARY: Changes in climate phenomena in the world have been intensely discussed in recent years, even generating divergent understandings of the real contemporary scenario. In this sense, the objective of this study was to evaluate the influence of lunar nodal cycles and periods of solar activities on the climatological behavior of Pernambuco cities. The work was carried out with precipitation and temperature data for Petrolina-PE and Recife-PE stations, obtained from the National Institute of Meteorology (INMET), for the period from 1961 to 2018. lunar nodal cycles and periods of 11 years for the Schwabe solar cycles, observing the variation of the described variables. The evaluations were performed through spreadsheets and generation of linear regressions and trend lines. It was observed that the cycles had no direct influence on the variables studied and that there were increases in temperatures and decreases in rainfall. Most of the trends in the cycles presented variations close to the trend of the total database.

Keywords: Petrolina. Recife. Temperature. Precipitation. Schwabe.

\section{INTRODUÇÃO}

As alterações dos fenômenos climáticos no mundo têm sido intensamente discutidas nos últimos anos. As elevadas temperaturas associadas a uma sequência anômala de anos secos têm gerado dificuldades para os habitantes do semiárido nordestino do Brasil (especialmente). Esta situação, além de inviabilizar ou minorar cultivos de sequeiro, vem gerando grandes especulações quanto ao aquecimento global do planeta. 
Já se apontam elevações na ordem de $0,85^{\circ} \mathrm{C}$ de aquecimento para os anos que separam os eventos contemporâneos aos pré-revolução industrial (Silva e Paula, 2009). Os relatórios do IPCC (Intergovernmental Panel on Climate Change - Painel Intergovernamental sobre Mudanças Climáticas) são os mais citados nestas constatações.

Registra-se também a existência de grupos de pesquisadores que se contrapõe aos relatórios do IPCC, porém, na grande maioria, apenas no tocante ao agente causador do aquecimento (antropogênico ou natural), sendo, quase unanimidade, para os aquecimentistas (termo empregado a quem defende o aquecimento antropogênico) e céticos (defendem o aquecimento do planeta por fatores naturais) que o planeta está se aquecendo. Apesar de não existir uma única fonte que cause estas alterações, já é constatado o aumento da temperatura em diversas partes do globo.

Os pesquisadores que defendem o aquecimento natural do planeta apontam diversas causas para tal fenômeno, dentre eles, Molion (2017) apresentou o ciclo nodal lunar, definido como o período em que a inclinação da lua em relação à terra varia de $18,4^{\circ}$ a $28,6^{\circ}$, aproximadamente bidecadal, como de potencial influência sobre a rotação geral da atmosfera e efeitos na formação de nuvens e precipitação, bem como no desencadear dos fenômenos El Niño e Lá Niña.

Também foi apontado por Molion (2005) o ciclo da atividade solar, período de cerca de 11 anos, em que acontece maior emissão de energia solar e em consequência ocorre aquecimento planetária. O autor aponta que este aquecimento natural da terra está sendo interpretado, erroneamente por muitos, como produto da emissão de gases e do efeito estufa.

Desta forma, ver-se claramente que análises dos elementos meteorológicos, temperatura e precipitação, em escala de tempo que envolva os períodos de manchas solares e ciclos nodais lunares podem propiciar novas interpretações sobre variações ou mudanças climáticas. Assim objetivou-se com este trabalho investigar a influência das fases nodais e ciclos solares de Schwabe no comportamento climatológico de temperatura e precipitação para cidades pernambucana.

\section{MATERIAL E MÉTODO}

O local de estudo foi selecionado devido a grande importância agronômica e a distribuição de estações para o Estado de Pernambuco, compreendendo Sertão, Petrolina-PE, e Litoral, Recife-PE (Figura 1). Foram utilizados dados históricos do INMET de precipitação e temperatura para as estações referentes ao período de 1961 a 2019 (INMET, 2019). 
Figura 1. Localização de estudo.

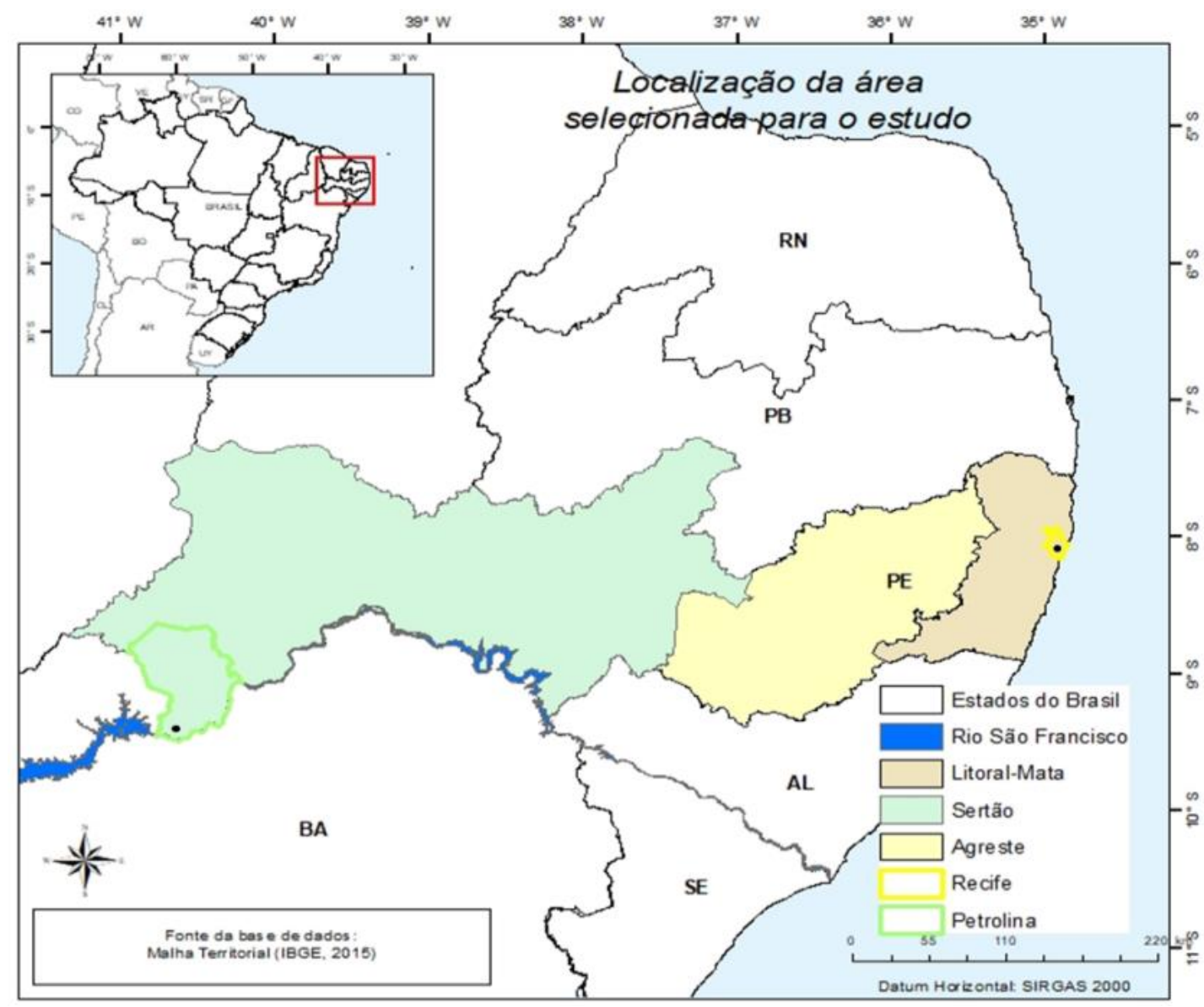

Fonte: Elaborado pelo Autor.

Para os ciclos nodais lunares, seguiu-se o entendimento de Lisitzin (1974), Mitra et al (1991) e Cook et al (1997) que descrevem que devido a Lua ter seu eixo de rotação inclinado em $5,1^{\circ}$ com relação ao plano da eclíptica e o da Terra ser de $23,5^{\circ}$, o movimento lunar ocorre na faixa latitudinal terrestre de $28,6^{\circ} \mathrm{N}$ a $28,6^{\circ} \mathrm{S}$ (isso em cerca de 20 anos), contudo, por cerca de 10 anos os eixos terra e lua encontram-se na mesma direção, e neste período o deslocamento da lua se concentra entre $18,4^{\circ} \mathrm{N}$ e $18,4^{\circ} \mathrm{S}$. Assim, os ciclos nodais lunares ocorrem em 20 anos divididos em períodos decadais com a lua movendo-se entre os trópicos e os outros 10 anos externo a estes.

Já para o ciclo de atividade solar, ou ciclo de Schwabe, considerou-se a indicação de Molion (2005) e Oliveira et al. (2018) com períodos de aproximadamente 11 anos. A observação desses ciclos iniciou-se em 1750, atualmente situado no ciclo 24 (ordem crescente desde o início do estudo dos ciclos).

Das séries temporais, os dados de temperatura mínima $\left(\operatorname{Tmin},{ }^{\circ} \mathrm{C}\right)$ e máxima $\left(\operatorname{Tmax},{ }^{\circ} \mathrm{C}\right) \mathrm{e}$ Precipitação Anual (mm) foram convertidos de diários para anuais, descartando os dias com 
falhas nas leituras, assim utilizados para verificação de tendência. A temperatura média foi obtida pelo método dos extremos (média entre as temperaturas máximas e mínimas).

As informações foram organizadas e tratadas em planilhas eletrônicas, sendo que as análises exploratórias de cada série temporal das variáveis climáticas (temperatura mínima, máxima, media e precipitação) foram realizadas a partir de regressões lineares com observância aos parâmetros das regressões e significâncias do $\mathrm{R}^{2}$.

\section{RESULTADO E DISCUSSÃO}

Na Figura 2.A pode se observar as linhas de tendência para precipitação, em forma decadal (ciclo nodal), normal climatológica (trinta anos), e série histórica total de dados da estação de Recife-PE. Pode-se perceber certas variações e comportamentos, como uma oscilação entre sequências de anos úmidos (excesso de precipitação) e anos secos (déficit de precipitação).

As análises dos ciclos nodais lunares demonstraram tendência ao decréscimo da precipitação, geralmente iniciando o ciclo com índices de pluviosidade mais elevados e ao decorrer do período ocorreu diminuição das chuvas. Os índices de pluviosidade variaram entre $1500 \mathrm{~mm}$ até $3800 \mathrm{~mm}$ por ano, demonstrando variação anual da precipitação, representando inconstância desse índice.

Com exceção ao padrão apresentado, onde a precipitação decresce ao decorrer dos anos, no período de 1980 a 1990 houve acréscimo nas precipitações (Figuras 2.A e 2.B), ocasionando uma linha de tendência crescente para essa década. Correspondente ao período do ciclo nodal no qual a lua está em fase de transição, de incorporada aos trópicos a externa aos trópicos, conforme relatado por Molion (2005).

Através da análise dos ciclos nodais lunares viabilizou-se a visualização das variações de precipitação que não seriam detectadas através da análise total dos dados (Molion, 2005), ou até mesmo através da normal climatológica. Essa variação visualizada nas Figuras 2.A e 2.D, correlacionada com o ciclo nodal não indicam sua influência direta na variação da precipitação, contrapondo-se em partes ao entendimento tecido por Molion (2017).

Observa-se ainda na Figura 2.E a 2.P para temperatura, crescentes valores de temperaturas mínimas, médias e máximas, com exceção do ciclo situado de 1970 a 1980. Fica evidente em maiores proporções quando observada à série histórica total. Esse aumento da temperatura poderá acarretar em variações negativas no balanço hídrico, devido a elevação do potencial evaporativo (Allen et al., 1998).

Esse trabalho corrobora com Lopes e Leal (2015) que observaram tendências de redução da precipitação para a cidade de Recife-PE com atenuação negativa de 2,3 mm para o período de 
1961 a 2014. Porém essa redução não ocasionou grandes alterações climáticas, levando em consideração que se trata de uma cidade litorânea, o oceano propicia a estabilidade climática da região.

Figura 2. Tendências de precipitação e temperatura para Recife-PE para os ciclos nodais lunares (A, I, E e M), ciclo solar de Schwabe (B, F, J e N), normais climatológicas (C, K, G e O) e série histórica (D, L, H e P).
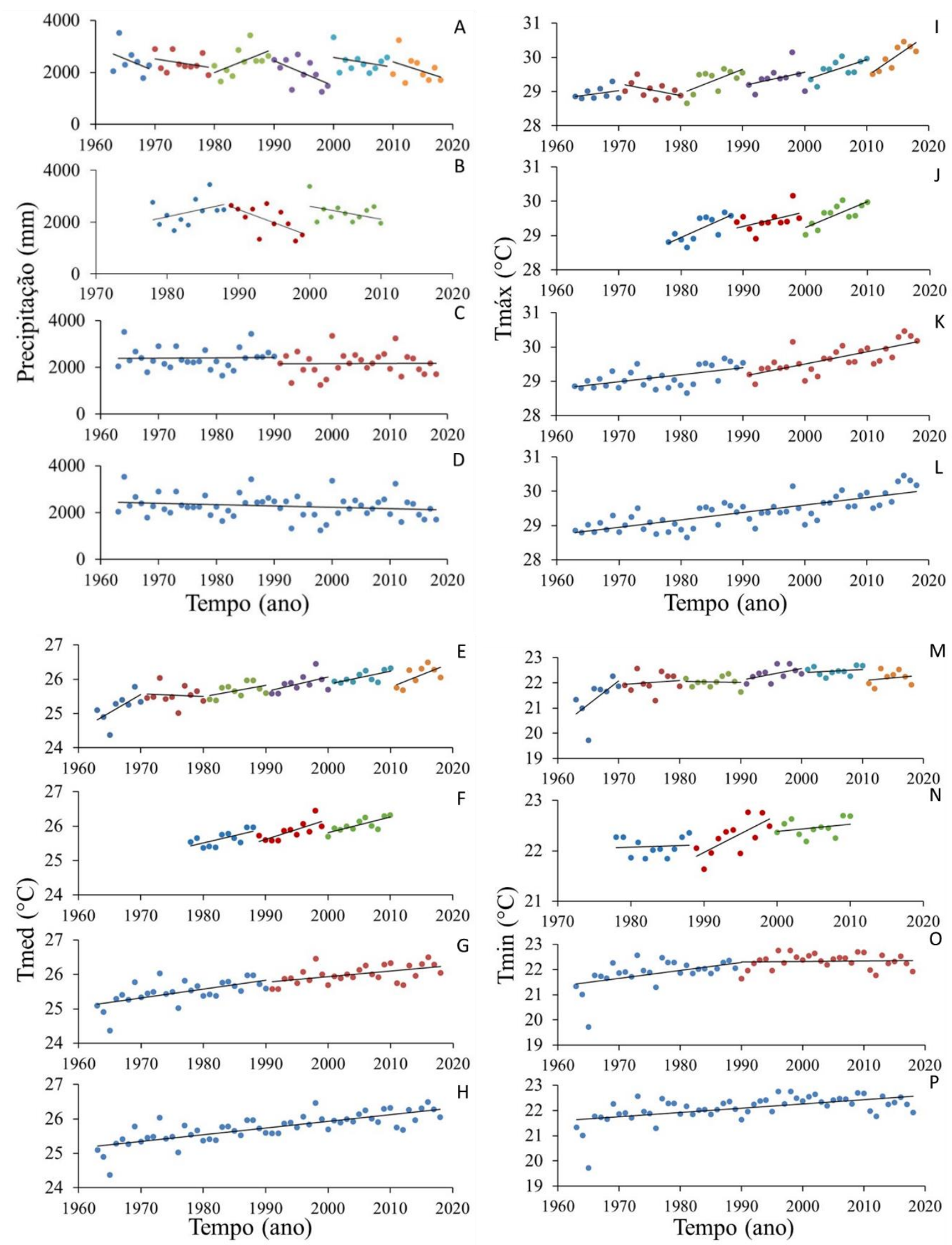

Fonte: Elaborado pelo Autor. 
Os ciclos solares de Schwabe relatados na Figura 2.B abordam os ciclos 21, 22 e 23, Leithold (2009) descreveu a redução das manchas solares ocorrida nesses ciclos. Porém, as tendências obtidas não demonstram representatividade para a variação do número de manchas solares, apresentando aumento da temperatura e redução na precipitação, semelhante a tendência da série histórica dos dados.

Tabela 1 - Parâmetros da equação linear $(\mathrm{Y}=\mathrm{bx}+\mathrm{a})$ para os diferentes ciclos nodais lunares, solares, normais climatológicas e série total de dados de 1960 a 2018 para a cidade de Recife-PE.

\begin{tabular}{|c|c|c|c|c|c|c|c|c|c|c|c|c|}
\hline \multicolumn{13}{|c|}{ Ciclo nodal } \\
\hline & \multicolumn{3}{|c|}{ Tmáx } & \multicolumn{3}{|c|}{ Tmed } & \multicolumn{3}{|c|}{ Tmin } & \multicolumn{3}{|c|}{$\mathrm{P}$} \\
\hline ID & $\mathrm{a}$ & $\mathrm{b}$ & $\overline{\mathrm{R}^{2}}$ & $\mathrm{a}$ & $\mathrm{b}$ & $\mathrm{R}^{2}$ & A & $\mathrm{b}$ & $\overline{\mathrm{R}^{2}}$ & $\mathrm{a}$ & $\mathrm{b}$ & $\mathrm{R}^{2}$ \\
\hline 1 & $-16,845$ & 0,0233 & 0,11 & $-182,14$ & 0,1054 & 0,39 & $-347,44$ & 0,1876 & 0,40 & 188036 & $-94,407$ & 0,13 \\
\hline 2 & 95,68 & $-0,0337$ & 0,20 & 40,672 & $-0,0077$ & 0,01 & $-14,336$ & 0,0184 & 0,01 & 72854 & $-35,699$ & 0,09 \\
\hline 3 & $-111,79$ & 0,0711 & 0,40 & $-111,79$ & 0,0711 & 0,24 & 30,492 & $-0,0043$ & 0,24 & -179413 & 91,623 & 0,30 \\
\hline 4 & 48,097 & 0,0388 & 0,12 & $-48,097$ & 0,0388 & 0,25 & $-70,708$ & 0,0466 & 0,25 & 195909 & $-97,218$ & 0,32 \\
\hline 5 & $-92,624$ & 0,061 & 0,44 & $-92,624$ & 0,061 & 0,45 & $-4,7584$ & 0,0136 & 0,45 & 78324 & $-37,876$ & 0,08 \\
\hline 6 & $-219,02$ & 0,1236 & 0,69 & $-219,02$ & 0,1236 & 0,40 & $-22,174$ & 0,022 & 0,39 & 151063 & $-73,952$ & 0,15 \\
\hline \multicolumn{13}{|c|}{ Ciclo solar Schwabe } \\
\hline & \multicolumn{3}{|c|}{ Tmáx } & \multicolumn{3}{|c|}{ Tmed } & \multicolumn{3}{|c|}{ Tmin } & \multicolumn{3}{|c|}{$\mathrm{P}$} \\
\hline ID & $\mathrm{a}$ & $\mathrm{b}$ & $\mathrm{r}^{2}$ & $\mathrm{a}$ & $\mathrm{b}$ & $\mathrm{R}^{2}$ & A & $\mathrm{b}$ & $\mathrm{R}^{2}$ & $\mathrm{a}$ & $\mathrm{b}$ & $\mathrm{R}^{2}$ \\
\hline 1 & $-133,16$ & 0,08 & 0,56 & $-59,95$ & 0,04 & 0,44 & 13,26 & 0,00 & 0,01 & -116941 & 60,17 & 0,15 \\
\hline 2 & $-56,51$ & 0,04 & 0,23 & $-91,18$ & 0,06 & 0,56 & $-125,84$ & 0,07 & 0,50 & 204560,00 & $-101,55$ & 0,41 \\
\hline 3 & $-121,24$ & 0,08 & 0,58 & $-64,20$ & 0,05 & 0,58 & $-7,15$ & 0,01 & 0,08 & 102021,00 & $-49,71$ & 0,17 \\
\hline \multicolumn{13}{|c|}{ Normal Climatológica } \\
\hline & \multicolumn{3}{|c|}{ Tmáx } & \multicolumn{3}{|c|}{ Tmed } & \multicolumn{3}{|c|}{ Tmin } & \multicolumn{3}{|c|}{$\mathrm{P}$} \\
\hline ID & $\mathrm{a}$ & $\mathrm{b}$ & $\mathrm{R}^{2}$ & $\mathrm{a}$ & $\mathrm{b}$ & $\mathrm{R}^{2}$ & A & $\mathrm{b}$ & $\mathrm{R}^{2}$ & $\mathrm{a}$ & $\mathrm{b}$ & $\mathrm{R}^{2}$ \\
\hline 1 & $-11,707$ & 0,0207 & 0,3168 & $-26,302$ & 0,0262 & 0,3723 & $-40,897$ & 0,0317 & 0,2285 & $-189,39$ & 1,3114 & 0,06 \\
\hline 2 & $-42,094$ & 0,0358 & 0,5396 & $-6,6413$ & 0,0163 & 0,2796 & 18,293 & 0,002 & 0,0035 & 960,66 & 0,6031 & 0,01 \\
\hline \multicolumn{13}{|c|}{ Série histórica } \\
\hline & \multicolumn{3}{|c|}{ Tmáx } & \multicolumn{3}{|c|}{ Tmed } & \multicolumn{3}{|c|}{ Tmin } & \multicolumn{3}{|c|}{$\mathrm{P}$} \\
\hline ID & $\mathrm{a}$ & $\mathrm{b}$ & $\mathrm{R}^{2}$ & $\mathrm{a}$ & $\mathrm{b}$ & $\mathrm{R}^{2}$ & A & $\mathrm{b}$ & $\mathrm{R}^{2}$ & $\mathrm{a}$ & $\mathrm{b}$ & $\mathrm{R}^{2}$ \\
\hline 1 & $-13,814$ & 0,0217 & 0,6273 & $-12,752$ & 0,0193 & 0,6119 & $-11,69$ & 0,017 & 0,314 & 14240 & $-6,0055$ & 0,041 \\
\hline
\end{tabular}

Onde: Tmáx, Tmed, Tmin e $\mathrm{P}$ - temperatura máxima, média, mínima do ar, ${ }^{\circ} \mathrm{C}$ e precipitação, $\mathrm{mm}$, respectivamente; ID - ciclo ou período de dados, apresentados em ordem crescente. Fonte: Elaborado pelo Autor.

Em estudos de correlação entre dados climáticos e produção agrícola realizados por Guimarães et al. (2016) foi observado que a agricultura não apresentou grandes variações na produção agrícola em respostas à diferentes regimes pluviométricos junto com variações da temperatura, demonstrando que apesar de condições variadas, a agricultura dispõe de fatores tecnológicos que ajudam a minimizar a variabilidade climática. Atrelado a esse comentário, pode- 
se inferir que o regime hídrico do Recife-PE continua com oferta regular com poucas variações ao longo dos anos.

Para o município de Petrolina, como já foi observado por Lopes et al. (2017), há limitação hídrica, ocasionada pelas elevadas temperaturas e baixos índices pluviométricos. Apesar das falhas de dados em diversos anos, observou-se tendência linear crescente para precipitação, com exceção da década atual (Figuras 3.A e 3.B) e do padrão de trinta anos (normal climatológica) e série total de dados (Figuras 3.C e 3.D). Em valores as precipitações variaram de 140 a $820 \mathrm{~mm}$ durante os anos estudados, com tendências divergentes entre os ciclos (lunares e solares) para as normais climatológicas e série histórica dos dados (comprovadas pelos coeficientes angulares, b, com predomínio de valores positivos para os ciclos nodais lunares e solares e negativos para as normais climatológicas e série histórica total, Tabela 2). 
Figura 3. Tendências de precipitação e temperatura para Petrolina-PE para os ciclos nodais lunares (A, I, E e M), ciclo solar de Schwabe (B, F, J e N), normais climatológicas (C, K, G e O) e série histórica $(\mathrm{D}, \mathrm{L}, \mathrm{H}$ e $\mathrm{P})$.
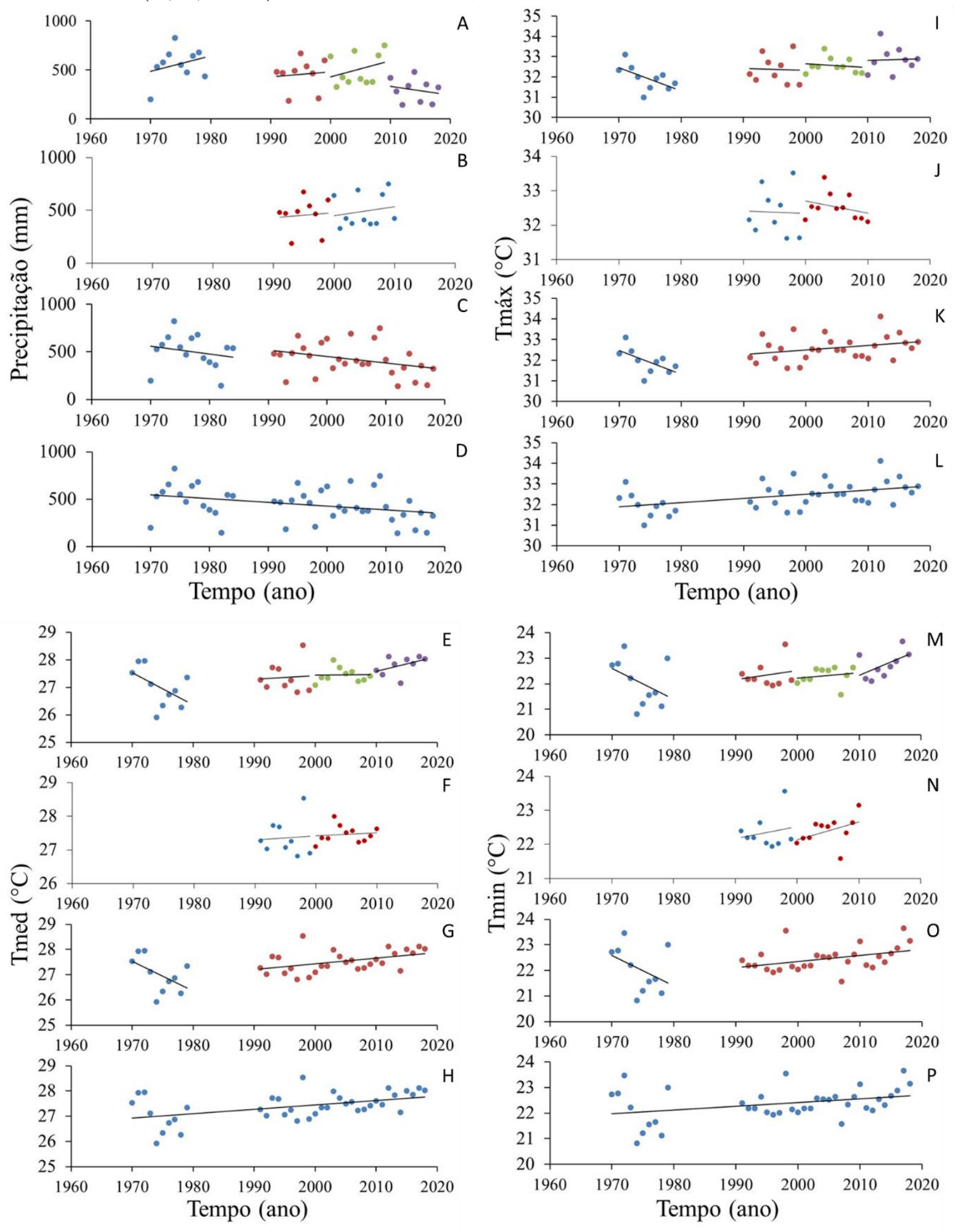

Fonte: Elaborado pelo Autor.

Ancorado nas análises dos dados apresentados é provável que não ocorra influência direta do ciclo nodal lunar e das atividades solares, nas estações analisadas para todas as variáveis temperatura e precipitação (Figuras 2 e 3; Tabelas 1 e 2). 
Tabela 2 - Parâmetros da equação linear $(\mathrm{Y}=\mathrm{bx}+\mathrm{a})$ para os diferentes ciclos nodais lunares, solares, normais climatológicas e série total de dados de 1970 a 2018 para a cidade de PetrolinaPE.

\begin{tabular}{|c|c|c|c|c|c|c|c|c|c|c|c|c|}
\hline \multicolumn{13}{|c|}{ Ciclo nodal } \\
\hline & \multicolumn{3}{|c|}{ Tmáx } & \multicolumn{3}{|c|}{ Tmed } & \multicolumn{3}{|c|}{ Tmin } & \multicolumn{3}{|c|}{$\mathrm{P}$} \\
\hline ID & $\mathrm{a}$ & $\mathrm{b}$ & $\mathrm{R}^{2}$ & $\mathrm{a}$ & $\mathrm{b}$ & $\mathrm{R}^{2}$ & $\mathrm{~A}$ & $\mathrm{~b}$ & $\mathrm{R}^{2}$ & $\mathrm{a}$ & $\mathrm{b}$ & $\mathrm{R}^{2}$ \\
\hline 1 & 259,47 & $-0,1152$ & 0,34 & 258,86 & $-0,1174$ & 0,01 & 258,26 & 0,1196 & 0,16 & -31302 & 16,135 & 0,0837 \\
\hline 2 & 49,006 & $-0,00833$ & 0,01 & 0,9891 & 0,0132 & 0,25 & $-47,028$ & 0,0348 & 0,03 & $-9489,9$ & 4,985 & 0,0072 \\
\hline 3 & 68,766 & $-0,0181$ & 0,02 & 24,177 & 0,0016 & 0,01 & $-20,412$ & 0,0213 & 0,04 & -32535 & 16,481 & 0,097 \\
\hline 4 & 17,348 & 0,0077 & 0,01 & $-81,399$ & 0,0542 & 0,21 & $-180,15$ & 0,1007 & 0,3 & 17844 & $-8,7133$ & 0,0393 \\
\hline \multicolumn{13}{|c|}{ Ciclo solar de Schwabe } \\
\hline & \multicolumn{3}{|c|}{ Tmáx } & \multicolumn{3}{|c|}{ Tmed } & \multicolumn{3}{|c|}{ Tmin } & \multicolumn{3}{|c|}{$\mathrm{P}$} \\
\hline ID & $\mathrm{a}$ & $\mathrm{b}$ & $\mathrm{R}^{2}$ & $\mathrm{a}$ & $\mathrm{b}$ & $\mathrm{R}^{2}$ & A & $\mathrm{b}$ & $\mathrm{R}^{2}$ & $\mathrm{a}$ & $\mathrm{b}$ & $\mathrm{R}^{2}$ \\
\hline 1 & 49,006 & $-0,0083$ & 0,0011 & 0,9891 & 0,0132 & 0,0045 & $-47,028$ & 0,0348 & 0,0362 & $-9489,9$ & 4,985 & 0,0072 \\
\hline 2 & 103,43 & $-0,0354$ & 0,0885 & 9,7272 & 0,0088 & 0,0134 & $-83,98$ & 0,0531 & 0,1893 & -16908 & 8,6791 & 0,035 \\
\hline \multicolumn{13}{|c|}{ Normal climatológica } \\
\hline & \multicolumn{3}{|c|}{ Tmáx } & \multicolumn{3}{|c|}{ Tmed } & \multicolumn{3}{|c|}{ Tmin } & \multicolumn{3}{|c|}{$\mathrm{P}$} \\
\hline ID & $\mathrm{a}$ & $\mathrm{b}$ & $\mathrm{R}^{2}$ & $\mathrm{a}$ & $\mathrm{b}$ & $\mathrm{R}^{2}$ & A & $\mathrm{b}$ & $\mathrm{R}^{2}$ & $\mathrm{a}$ & $\mathrm{b}$ & $\mathrm{R}^{2}$ \\
\hline 1 & 259,47 & $-0,1152$ & 0,3408 & 258,86 & $-0,1174$ & 0,2559 & 258,86 & $-0,1174$ & 0,2559 & 16234 & $-7,9575$ & 0,0394 \\
\hline 2 & $-11,074$ & 0,0218 & 0,0908 & $-18,412$ & 0,0229 & 0,199 & $-25,75$ & 0,0241 & 0,1722 & 14153 & $-6,8508$ & 0,1114 \\
\hline \multicolumn{13}{|c|}{ Serie histórica } \\
\hline & \multicolumn{3}{|c|}{ Tmáx } & \multicolumn{3}{|c|}{ Tmed } & \multicolumn{3}{|c|}{ Tmin } & \multicolumn{3}{|c|}{$\mathrm{P}$} \\
\hline ID & $\mathrm{a}$ & $\mathrm{b}$ & $\mathrm{R}^{2}$ & $\mathrm{a}$ & $\mathrm{b}$ & $\mathrm{R}^{2}$ & A & $\mathrm{b}$ & $\mathrm{R}^{2}$ & $\mathrm{a}$ & $\mathrm{b}$ & $\mathrm{R}^{2}$ \\
\hline 1 & $-8,3682$ & 0,0204 & 0,2242 & $-7,5184$ & 0,0175 & 0,2303 & $-6,6687$ & 0,0145 & 0,1224 & 8203,2 & $-3,8871$ & 0,1115 \\
\hline
\end{tabular}

Onde: Tmáx, Tmed, Tmin e $\mathrm{P}$ - temperatura máxima, média, mínima do ar, ${ }^{\circ} \mathrm{C}$ e precipitação, $\mathrm{mm}$, respectivamente; ID - ciclo ou período de dados, apresentados em ordem crescente.

Em estudo anterior foi observado por Leithold (2009) que o ciclo 23, situado na linha do equador, apresenta menores efeitos do geomagnetismo, sendo explicado pelo número de manchas, que a partir de 2006 as atividades solares reduziram o número de manchas. Contudo as equações obtidas através das linhas de tendência, apresentadas na Tabela 2, não corroboram com o padrão apresentado pelo autor acima citado, indicando aumento da temperatura e precipitação, sem a variação apresentada pela atividade solar.

\section{CONCLUSÃO}

Os fenômenos climáticos recorrentes nos últimos anos indicam predomínio de acentuação da temperatura para todos os ciclos estudados no litoral e sertão pernambucano e redução da precipitação apenas para as normais climatológicas e série histórica total. 
As fases nodais e ciclos solares não apresentaram representatividade para as variações climáticas das duas estações pernambucanas estudadas.

\section{REFERÊNCIAS}

ALLEN, R. G.; PEREIRA, L. S.; RAES, D.; SMITH, M. Crop evapotranspiration - Guidelines for computing crop water requirements. Roma: FAO Irrigation and Drainage, v. 56, 1998. P.297.

COOK, E. R.; MEKO, D. M.; STOCKON, C. W. A new assessment of possible solar and lunar forcing of the bidacadal drought rhythm in the western United States. Journal of Climate, v.10, n.6, p. 1343-1356, 1997.

INMET. Instituto Nacional de Meteorologia. Previsão do tempo. Disponível em: $<$ http://www.inmet.gov.br/portal/index.php?r=home/page\&page=sm_previsao_tempo $>$ acesso em: 18 de set. 2019 a.

INMET. Instituto Nacional de Meteorologia. Sobre o INMET. Disponível em:

<http://www.inmet.gov.br/portal/index.php? $\mathrm{r}=$ home/page\&page=sobre_inmet $>$ acesso em: $18 \mathrm{de}$ set. $2019 \mathrm{~b}$.

GUIMARAES, M. J. M.; LOPES, I.; OLDONI, H.; COELHO FILHO, M. A. Balanço hídrico para diferentes regimes pluviométricos na região de Cruz das Almas-BA. Revista de ciências agrárias, v. 59, p. 252-258, 2016.

LEITHOLD, Â. A. Ciclo Solar de Schwabe, o Ciclo 24 e possibilidades de previsão de seu comportamento futuro. Relatório de pesquisa, 2009.

LOPES, I.; LEAL, B. G. Índice de aridez e tendência a desertificação para estações meteorológicas nos estados da Bahia e Pernambuco. Revista brasileira de climatologia, v. 17, p. 155-172, 2015.

LOPES, I.; GUIMARAES, M. J. M.; MELO, J. M. M.; RAMOS, C. M. C. Balanço hídrico em função de regimes pluviométricos na região de Petrolina-PE. Irriga, v. 22, p. 443, 2018.

MITRA, K.; MUKHERJI, S.; DUTTA, S. N. Some indications of 18.6 year LUNI-Solar and 1011 year solar cycles in rainfall in North-West India, the plains of Uttar Pradesh and North-Central India. Journal of Geophysical Research: Oceans, v.112, n.2, 2007.

MOLION, L. C. B. Aquecimento global, El Niños, manchas solares, vulcões e Oscilação Decadal do Pacífico. Climanálise, v.1, n.3, 2005.

MOLION L. C. B. Gênese do El Niño. Revista Brasileira de Climatologia, v.21: p.1-4. 2017.

OLIVEIRA, M. J.; CARNEIRO, C. D. R.; VECCHIA, F. A. da S.; BAPTISTA, G. M. de M. Ciclos climáticos e causas naturais das mudanças do clima. Terrae Didatica, v.13, n.3, p.149184. 2018.

SILVA, R. W. C., PAULA, B. L. Causa do aquecimento global: antropogênica versus natural. Terræ Didatica, v. 05, n. 01, p. 42-49, 2009. 
